\title{
Competition with Licensed Shared Spectrum
}

\author{
Chang Liu \\ EECS Department \\ Northwestern University, Evanston, IL 60208 \\ Email: changliu2012@u.northwestern.edu
}

\author{
Randall A. Berry \\ EECS Department \\ Northwestern University, Evanston, IL 60208 \\ Email: rberry@eecs.northwestern.edu
}

\begin{abstract}
This paper considers sharing wireless spectrum via licensed secondary access in which a single firm has an exclusive license for secondary use of a spectrum band. Building on previous work modeling competition among wireless service providers, we study competition between primary and secondary firms with this type of sharing, and characterize its impact on social welfare, consumer welfare and firm profits.
\end{abstract}

\section{INTRODUCTION}

The rising demand for wireless data and the limited supply of wireless spectrum has fueled interest in novel approaches for spectrum sharing. Indeed, sharing has been adopted in the TV white spaces [1] and is being considered for federal spectrum such as $3.5 \mathrm{GHz}$ band [2]. Many different approaches to spectrum sharing have been studied, e.g., [3]-[5], much of this under the "primary-secondary" model. Here, the primary user has priority usage of the frequency band, while the secondary users are allowed to access the spectrum provided that they do not degrade the primary's service. We consider a similar primary-secondary sharing model as in [5], where both primary and secondary users are wireless service providers (SPs) who compete for a common pool of customers.

Our approach is an extension of work in [6]-[8] modeling competition among SPs. This was in turn used models for price competition with congestible resources developed in the operations and economics literature (e.g. [9], [10]). In these models, SPs set prices to compete for the same pool of customers, and customers choose SPs based on the delivered price given by the sum of a SP's price and a congestion cost. In [6], we considered such a model for sharing the band of a single primary SP with multiple secondary SPs. The assumption was that secondary use was unlicensed, i.e., any secondary firm could use the band provided it did not increase the congestion cost experience by the primary SP's customers. However, the customers of each secondary SP experienced congestion due to both the traffic from all the secondary SPs and the traffic of the primary SP.

The results in [6] show that due to competition among multiple secondary SPs, the equilibrium price charged by each secondary SP is zero. Allowing for such secondary SPs does result in more customers being served and, moreover, both social welfare and consumer welfare increase compared to no sharing, provided there is sufficient bandwidth available, while the primary firm's profit decreases. This makes sharing

This research is supported in part by NSF grants CNS-1147786, SES1247984 and SES-1343381. undesirable for the primary. Also, since the secondary SPs make no profit, they would have little incentive to invest in offering service. Instead of unlicensed sharing, here we consider a scenario where a single secondary SP is licensed to operate in the same band, again provided that it will not cause any interference for the primary users. With this sharing scheme, we show that social welfare and consumer welfare still increase compared to without sharing. More interestingly, total firm profits are greater than with unlicensed sharing and in some regimes, greater than with no sharing.

The rest of the paper is organized as follows. Our model for price competition is described in Section II. We analyze the welfare under different sharing regimes and assumptions on customer demand and the congestion costs in Sections III and IV. Finally, we conclude in Section V.

\section{Competition Model}

As in [6]-[8], we consider a model where SPs set prices for their service to compete for a common pool of customers. As in [6], we focus on a model in which there is one incumbent SP with licensed spectrum. Without sharing, this incumbent acts as a monopolist with exclusive use of the frequency band. In the sharing model in [6], this incumbent is the primary user while a set of $N>1$ secondary SPs operate in the same band subject to not degrading the performance seen by the primary users. Here, we instead consider a model for licensed secondary sharing, where only one secondary SP is allowed to access the spectrum.

Each SP's service is characterized by a congestion cost, $g(x)$, which models various effects such as increased interference or queueing delays that arise as a SP serves more customers in a given area. With sharing, the congestion cost seen by the primary users is given by $g\left(x_{1}\right)$, where $x_{1}$ denotes the mass of customers the primary SP is serving. The congestion cost of the secondary SP's customers is given by $g(X)$, where

$$
X=x_{1}+x^{S}
$$

Here, $X$ is the total mass of customers being served in the band, and $x^{S}$ is the mass of customers served by the secondary SP. This models the fact that the primary SP does not "see" any degradation from the secondary, while the secondary users do experience degradation due to the primary. In general, we assume that $g(x)$ is an increasing, convex function as shown in Figure 1, though for simplicity we first focus on the case where 
this is a linear function, i.e., $g(x)=\frac{x}{C}$, where $C$ represents the bandwidth or capacity of the spectrum band.

Customers select SPs based on the SP's delivered price, which is the sum of the SP's announced price and the congestion cost of their service. Specifically, the delivered price of the primary SP is $p_{1}+g\left(x_{1}\right)$, while the delivered price of the secondary SP is $p_{2}+g(X)$. Each customer selects the service provider who has the lowest delivered price.

We also assume a single mass of infinitesimal customers. Customer demand is modeled by an inverse demand function $P(q)$, which will be a non-increasing function, that indicates the delivered price at which $q$ customers are willing to pay for service. As in Fig. 1, this will in general be a concave function. However, for simplicity, we first assume that customers are homogeneous in their demand, i.e., all customers are willing to pay up to the same delivered price, in which case $P(q)$ has a "box" shape.

We view the competition among the primary firm, the secondary as well as the customers as a pricing game where firms simultaneously set prices, $\left(p_{1}, p_{2}\right)$. Customers then choose one of the firms offering the lowest delivered price. Thus given a set of prices, $\left(p_{1}, p_{2}\right)$, the customers selecting service, must satisfy the following Wardrop equilibrium conditions [11]:

$$
\begin{aligned}
\left.p_{1}+g\left(x_{1}\right) \leq P(X), \quad \text { (with equality if } x_{1}>0\right) . \\
\left.p_{2}+g(X) \geq P(X), \quad \text { (with equality if } x_{2}>0\right) .
\end{aligned}
$$

These conditions specify that the delivered price of all SPs serving customers are equal and no greater than $P(X)$.

We define a (pure strategy) Nash equilibrium of the overall pricing game to be a set of prices $\left(p_{1}, p_{2}\right)$ and demands $\left(x_{1}, x_{2}\right)$ which satisfy these Wardrop equilibrium conditions and also have the property that no SP can increase its profit (given by $p_{i} x_{i}$ ) by unilaterally changing its price.

Given such an equilibrium, the firm profit, $f_{c}$, is defined by the sum of the profits made by both SPs. The welfare of the $x$ th customer served is the difference between that customer's value for the service given by $P(x)$ and the delivered price it pays for service; customers that are not served receive zero welfare. The total consumer welfare, $S_{c}$, is the integral of this over all customers. The social welfare, $S$, of the entire economy is the sum of the firm profit and the consumer welfare, i.e., $S=S_{c}+f_{c}$.

Next we briefly discuss the optimization faced by the primary firm with no sharing and unlicensed sharing and then contrast this with the case of licensed sharing.

\section{A. Without Sharing}

Without spectrum sharing, the primary firm is a monopolist in the market. As there is no competition with other firms, it sets the price to maximize its own profit, i.e., it solves:

$$
\begin{array}{ll}
\max & p x \\
\text { subject to } & p+g(x)=P(x), \\
& 0 \leq x \leq 1 .
\end{array}
$$

Under our assumptions, this will be a convex problem with a unique solution.

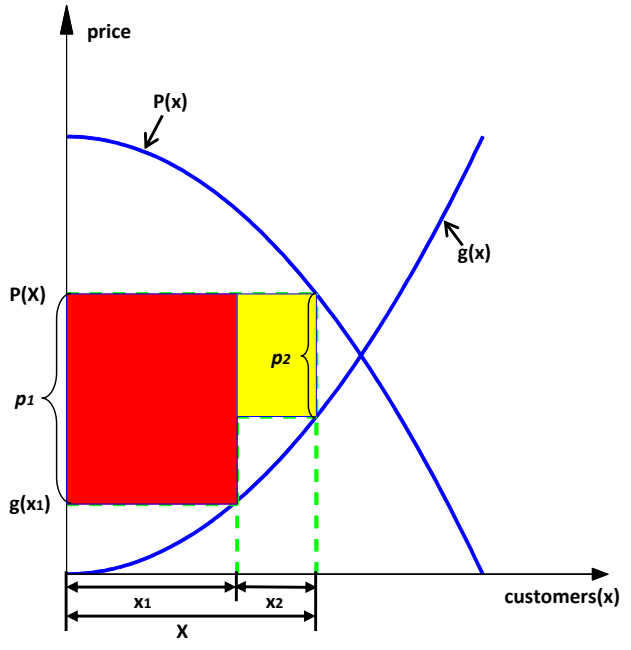

Fig. 1. Illustration of pricing game between primary and secondary firms with licensed shared spectrum.

\section{B. With Unlicensed Sharing}

With unlicensed sharing, it is shown in [6] that all the secondary firms have equilibrium price $p=0$. Thus, the delivered price is determined by $P\left(x^{*}\right)$, which satisfies $g\left(x^{*}\right)=P\left(x^{*}\right)$. Consequently, the primary firm needs to maximize its profits according to the fixed delivered price, thus it solves:

$$
\begin{array}{ll}
\max & p x \\
\text { subject to } & p+g(x)=P\left(x^{*}\right), \\
& 0 \leq x \leq x^{*} .
\end{array}
$$

Note the only difference between unlicensed sharing and no sharing is that the price cap of the former is fixed by (??) while the latter is given by $P\left(x^{*}\right)$.

\section{With Licensed Sharing}

With unlicensed sharing, the strategic interaction between the primary and secondary SPs is only via the fixed price cap $P\left(x^{*}\right)$. However, with licensed sharing, the secondary SP can sustain a non-zero price, and thus there is greater strategic interaction. In particular, though both firms must have the same delivered price in equilibrium, the value of this price is no longer fixed a priori but requires finding an equilibrium of the pricing game between the two providers. Such an equilibrium is characterized as a fixed point of the provider's best response correspondences, $B r_{i}\left(p_{-i}\right)$, which, for $i=1,2$, specify SP $i$ 's optimal price $p_{i}^{*}$ given that the other SP's price is fixed at $p_{-i}$. For the primary $\operatorname{SP}(i=1)$, this is given by solving:

$$
\begin{array}{ll}
\max & p_{1} x_{1} \\
\text { subject to } & p_{1}+g\left(x_{1}\right)=P(X), \\
& p_{2}+g(X)=P(X), \\
& x_{1}+x_{2}=X, \\
& 0 \leq x_{1}, x_{2} \leq 1,
\end{array}
$$

where $p_{2}$ is fixed and the optimization is over $p_{1}, x_{1}, x_{2}$ and $X$. The secondary SP solves the same problem, except the 


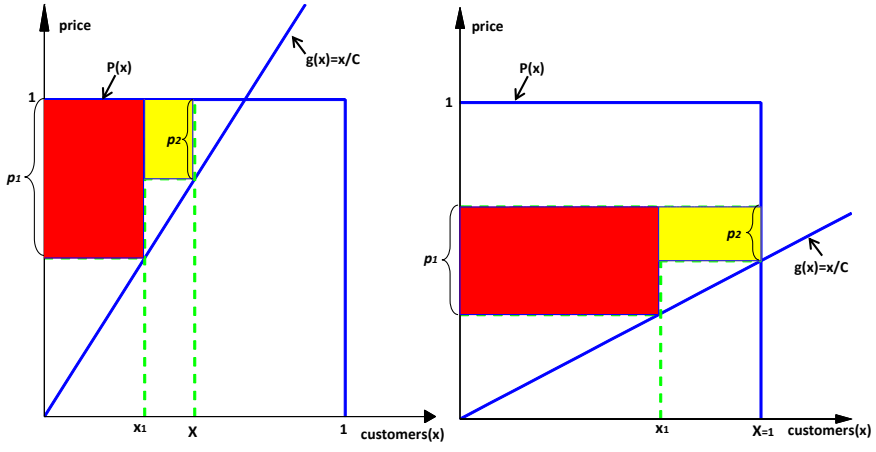

Fig. 2. Illustration of pricing game with linear congestion and homogeneous inverse-demand. Left: $C \leq 4 / 3$. Right: $C>4 / 3$.

objective is replaced by $p_{2} x_{2}$ and $p_{2}$ is fixed instead of $p_{1}$.

\section{Linear Congestion And Homogeneous Demand}

In this section, we assume a linear congestion cost given by $g(x)=x / C$ and a homogeneous customer demand given by

$$
P(x)= \begin{cases}1, & 0 \leq x \leq 1, \\ 0, & \text { otherwise }\end{cases}
$$

Later we will relax the width and height of this "box". The main result is summarized in the following theorem.

Theorem 3.1: When $0<C \leq 1.2$, with licensed sharing, social welfare is greater than that without sharing and with unlicensed sharing. When $C>1.2$, it asymptotically approaches to but is less than that with unlicensed sharing.

When $0<C \leq 1.49$, with licensed sharing, firm profit is greater than without sharing and with unlicensed sharing. When $C>1.49$, it drops below that without sharing and asymptotically approaches to but is greater than with unlicensed sharing.

With licensed sharing, consumer welfare is always no greater than that with unlicensed sharing and no less than without sharing.

The proof of this follows from comparing the equilibrium welfares under licensed sharing given in the following lemma to those for unlicensed sharing and no sharing, given in [6].

Lemma 3.2: With licensed spectrum sharing, under the assumed homogeneous demand and linear congestion cost there is always a unique Nash equilibrium with the following properties:

- When $0<C<4 / 3$, the primary SP serves $x_{1}=C / 2$ customers with $p_{1}=1 / 2$ and the secondary SP serves $x_{2}=C / 4$ customers with $p_{2}=1 / 4$ resulting in $S=$ $5 C / 16, f_{c}=5 C / 16$, and $S c=0$.

- When $C \geq 4 / 3$, the primary SP serves $x_{1}=2 / 3$ customers with $p_{1}=2 /(3 C)$ and the secondary SP serves $x_{2}=1 / 3$ customers with $p_{2}=1 /(3 C)$ resulting in $S=1-7 / 9 C, f_{c}=5 / 9 C$, and $S_{c}=1-4 / 3 C$.

This lemma shows that the possible outcomes are divided into two distinct cases illustrated in Fig. 2. The left-hand side of this figure illustrates the first class of equilibria in which spectrum is sufficiently limited so that even with sharing, all customers are not served $(C<4 / 3)$. In this case the delivered price is equal to the maximum value acceptable by consumers (i.e., $P(x)=1$ ). In the second case, shown on the righthand side of Fig. 2, there is enough spectrum available so that the entire market is served in equilibrium. In this case, price competition between the service providers lowers the delivered price below $P(x)=1$.

Proof: To begin, we consider the secondary SP and determine its best response to an arbitrary price $p_{1}<1 .^{1}$ This is given by solving

$$
\begin{array}{ll}
\max & p_{2} x_{2} \\
\text { subject to } & p_{2}+X / C \leq 1, \\
& p_{1}+x_{1} / C=p_{2}+X / C, \\
& x_{1}+x_{2}=X, \\
& 0 \leq X \leq 1, \\
& x_{1} \geq 0, x_{2} \geq 0 .
\end{array}
$$

Where the second constraint follows from the Wardrop equilibrium conditions and the fact that for the secondary to serve any customers and make a positive profit, this condition must be met with equality. From this constraint, we obtain $p_{1}+x_{1} / C=p_{2}+\left(x_{1}+x_{2}\right) / C$, which yields $x_{2}=C\left(p_{1}-p_{2}\right)$. Substituting this into the objective, it can be seen that the resulting convex problem has the unique solution

$$
p_{2}=p_{1} / 2,
$$

i.e., the secondary firm's best response is always to announce half of the price of the primary firm. This is true regardless of the amount of spectrum $C$.

Next we turn to the primary SP, its best response is given by solving

$$
\begin{array}{ll}
\max & p_{1} x_{1} \\
\text { subject to } & p_{1}+x_{1} / C \leq 1 \\
& p_{1}+x_{1} / C \leq p_{2}+X / C \\
& x_{1}+x_{2}=X, \\
& 0 \leq X \leq 1, \\
& x_{1} \geq 0, x_{2} \geq 0 .
\end{array}
$$

Note that at least one of the first two constraints must be tight at any optimal solution, since otherwise $p_{1}$ can be increased keeping all other parameters fixed yielding higher profit. Further, if the second constraint is tight and the first one is not, then it must be that $X=1$, or else $x_{1}$ could be increased, again leading to higher profit. In other words, the primary SP's best response will always result in either the delivered price being equal to the maximum acceptable value $(P(x)=1)$ or the entire market being served $(X=1)$.

Consider the case where the first constraint is binding, but not the second. In this case the primary's profit maximizing

\footnotetext{
${ }^{1}$ If $p_{1} \geq 1$, the primary will not serve any customers, since the delivered price would then be greater than 1 .
} 
price must be the same as in a market without sharing, in which case it solves

$$
\begin{array}{ll}
\max & p_{1} x_{1} \\
\text { subject to } & p_{1}+x_{1} / C=1, \\
& 0 \leq x_{1} \leq 0,
\end{array}
$$

As shown in [6], for $C \leq 2$, the solution to this problem is to set $p_{1}=1 / 2$ and serve $x_{1}=C / 2$ customers. For $C$ in this range, with licensed sharing, we must also ensure that the second constraint is satisfied, i.e., that

$$
p_{1}+x_{1} / C \leq p_{2}+X / C .
$$

Provided that $p_{2}+X / C \geq 1$ this constraint will be satisfied. Further, as discussed previously, if this constraint is binding and the first constraint is not, it must be that $X=1$; hence a necessary and sufficient condition for this constraint to be satisfied at a solution to (2) is if $p_{2} \geq 1-\frac{1}{C}$. Hence, we have shown that for $C \leq 2$, the primary SP's best response to any $p_{2} \geq 1-\frac{1}{C}$ is the same as in the no-sharing case, i.e., $p_{1}=\frac{1}{2}$. Combining this with our earlier observation that the secondary SP's best response is always $p_{2}=p_{1} / 2$, it follows that $p_{1}=1 / 2$ and $p_{2}=1 / 4$ is a Nash equilibrium if and only if $1 / 4 \geq 1-\frac{1}{C}$ or equivalently $C \leq 4 / 3$.

At any other Nash equilibria, it must be that (3) is the tight and the delivered price is less than 1 , which from the above means that either $C>4 / 3$ or $p_{2}<1-\frac{1}{C}$. Recall, that (3) being tight is equivalent to

$$
x_{2}=\left(p_{1}-p_{2}\right) C,
$$

in which case, the primary's best response is equivalent to solving

$$
\begin{array}{ll}
\max & p_{1} x_{1} \\
\text { subject to } & x_{1}+\left(p_{1}-p_{2}\right) C \leq 1 \\
& x_{1} \geq 0 \geq 0 .
\end{array}
$$

The unique solution to this problem is $p_{1}=\left(1-p_{2} C\right) /(2 C)$ for $p_{2}<1 / C$ and $p_{1}=p_{2}$, otherwise. Combining this with the secondary's best response of $p_{2}=p_{1} / 2$, yields the unique solution: $p_{1}=2 /(3 C)$ and $p_{2}=1 /(3 C)$. Furthermore, it can be seen that for $C<4 / 3$, this value of $p_{2}$ can not be smaller that $1-1 / C$, showing that this equilibrium arises if and only if $C \geq 4 / 3$ (when $C=4 / 3$ it corresponds to the previous equilibrium).

The number of customers served and the welfares for each equilibrium then follow by direct calculation.

From Lemma 3.2 it can be seen that when $C<4 / 3$, firm profits increase with the available bandwidth, since it allows them to serve more customers, while holding their price fixed. When $C>4 / 3$, firm profits are decreasing in $C$. In this case they continue serving the same mass of customers but at lower prices. As shown in Fig. 3-4 the loss of firm profit is more than offset by an increase in consumer welfare, resulting in an overall increase in social welfare.

In Fig. 3-4, we also show the welfares obtained under unlicensed sharing and without sharing, both of which are

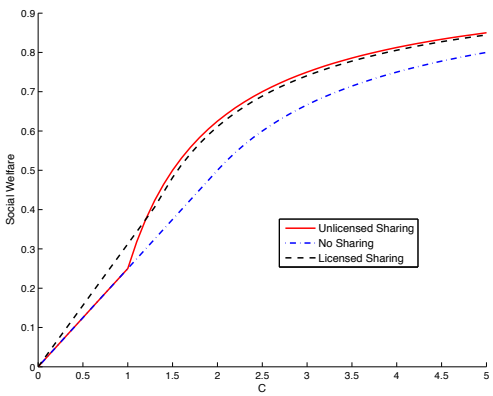

Fig. 3. Comparison of social welfare between unlicensed sharing, no sharing and licensed sharing with linear congestion and homogeneous inverse-demand.
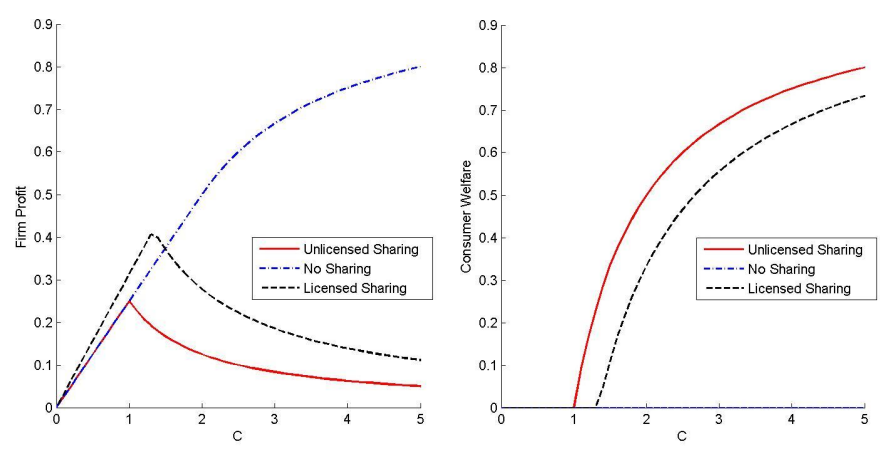

Fig. 4. Comparison of firm profit (left) and consumer welfare (right) between unlicensed sharing, no sharing and licensed sharing with linear congestion and homogeneous inverse-demand.

studied in [6]. With no sharing, the primary SP acts as a monopolist and extracts all of the welfare (i.e., consumer welfare is zero). With unlicensed sharing, the primary SP can no longer do this, when there is sufficient spectrum available, leading to increased consumer welfare and increased social welfare, although firm profits may decrease. The behavior is somewhat similar with licensed sharing, namely, with sufficient spectrum, consumer welfare increases and firm profits decreases relative to no sharing. The gain in consumer welfare is smaller than with unlicensed sharing. However, unlike unlicensed, with $C$ is small, licensed sharing results in an increase in overall welfare relative to no sharing (and unlicensed sharing). This is due to the additional firm profit gained by the secondary SP. However, when $C$ becomes large enough, i.e., $C>1.2$, the social welfare of licensed sharing still increases with bandwidth but drops below unlicensed sharing. This is because the competition between the two SPs does not increase consumer welfare as much as with unlicensed sharing.

So far we have investigated the welfares under the linear congestion cost and unit "box" demand function. Next we consider the general case of homogeneous demand, where both the width and the height of the "box" can be arbitrary constants $a$ and $b$, respectively. That is,

$$
P(x)= \begin{cases}b, & 0 \leq x \leq a \\ 0, & \text { otherwise }\end{cases}
$$


Following a similar analysis, we obtain a generalization of Lemma 3.2, which is given next.

Lemma 3.3: With licensed spectrum sharing, under general homogeneous demand and linear congestion cost there is always a unique Nash equilibrium with the following properties:

- When $0<C \leq 4 a / 3 b$, the primary SP serves $x_{1}=$ $b C / 2$ customers with $p_{1}=b / 2$ and the secondary SP serves $x_{2}=b C / 4$ customers with $p_{2}=b / 4$ resulting in $S=\frac{5 b^{2}}{16}, f_{c}=\frac{5 b^{2} C}{16}$, and $S c=0$.

- When $C>4 a / 3 b$, the primary SP serves $x_{1}=2 a / 3$ customers with $p_{1}=2 a / 3 C$ and the secondary SP serves $x_{2}=a / 3$ customers with $p_{2}=a / 3 C$ resulting in $S=$ $a b-\frac{7 a^{2}}{9 C}, f_{c}=\frac{5 a^{2}}{9 C}$, and $S_{c}=a b-\frac{4 a^{2}}{3 C}$.

A detailed comparison of the results in this lemma to the cases of unlicensed secondary sharing and no sharing are given in the top part of Table I. For all choices of $a$ and $b$, licensed sharing still generates the largest social welfare when $C$ is small. When $C$ grows larger, the social welfare of licensed sharing drops below that with unlicensed sharing with a difference of $a^{2} / 36 C$, which vanishes as $C$ becomes large. Also the crossing point between the two curves occurs when $C=1.2 a / b$. For the firm profit, licensed sharing performs best with limited bandwidth. However, the firm profit under licensed sharing decreases fast when the two SPs are able to serve the entire market. This is dramatically different from the case without sharing, where firm profit always increases monotonically with $C$. The crossing point of firm profit with licensed sharing and without sharing is occurs when $C=1.49 C$, and the difference between them can be as large as $b^{2} C / 16$.

\section{Heterogeneous Demand And General CONGESTION}

In this section we turn to a case with heterogeneous demand modeled by allowing the inverse demand to be any strictly decreasing, concave function and also allow the congestion cost is any strictly increasing convex function. The following theorem shows that with the additional assumption that $P(x)$ and $g(x)$ are twice differentiable, then a Nash equilibrium always exists for this game.

Theorem 4.1: Let the congestion function $g(x)$ be convex and increasing in $x$, and inverse demand function $P(x)$ be concave and decreasing, with both twice differentiable and $P(0)>0$. Then a Nash equilibrium exists under licensed sharing.

We omit the proof due to space considerations. Our next results shows that some of the qualitative behavior of social welfare and consumer welfare with homogeneous demands and linear congestion carry over to this more general setting.

Theorem 4.2: Given increasing convex congestion cost and decreasing concave inverse-demand, the equilibrium social welfare as well as consumer welfare with licensed sharing is always greater than that without sharing.

Proof: First note that due to the introduction of competition, the delivered price for licensed sharing will always be less than that without sharing. This follows since if this was not true the primary could announce the same price as without sharing and would be able to serve the same number of users. Thus, referring to Fig. 1, the area bounded by the delivered price of licensed sharing and $P(X)$, which equals the consumer welfare of licensed sharing, is larger than the consumer welfare without sharing plus the partial firm profit without sharing in that region and so is clearly larger than consumer welfare without sharing. In addition, the red shaded rectangle in Fig. 1 must have the largest area of any rectangle within the region bound by the delivered price and the congestion (since this area corresponds to the profit obtained by the primary firm with licensed sharing). In particular, this area must be no smaller than the other part of firm profit from without sharing. Only adding up the consumer welfare and the primary firm's profit with licensed sharing exceeds the social welfare of no sharing. Thus social welfares with licensed sharing is also larger than that without sharing.

To further explore the heterogeneous demand scenario, we consider a case with linear congestion cost $g(x)=x / C$ and linear inverse-demand $P(x)=b-b x / a$ with $0<x \leq a$ and $P(x)=0$, otherwise. In this case the equilibrium welfares can be calculated explicitly and are given in the lower part of Table I, along with the corresponding quantities for unlicensed sharing and no sharing. In this setting, the ratio of social welfare in licensed sharing to unlicensed sharing is shown to be about $5 / 4$ when $C$ is small. When $C$ becomes large, the ratio becomes less than but approaches to 1 . The crossing point always exists. In comparison, the ratio of firm profit with licensed sharing to no sharing is approximately $5 / 4$ as well when $C$ is small. And as $C$ becomes large, the ratio approaches to zero. To further illustrate this behavior, we also plot these quantities as a function of $C$ in Fig. 5, assuming that $a=b=1$.

The next theorem compares the primary firm's profit with unlicensed sharing and licensed sharing.

Theorem 4.3: Given strictly increasing and convex congestion and strictly decreasing and concave inverse-demand, the primary firm gains more profit and serves more customers with licensed sharing than with unlicensed sharing.

Proof: Let $P(X)$ be the delivered price with licensed sharing; this will be strictly greater than the delivered price with unlicensed sharing $P\left(X^{*}\right)$, since if not the secondary SP would not make any positive profit. From this it follows directly that the primary firms' profit must be greater with licensed sharing. TO complete the proof we only need to show that $x_{1}^{*}<x_{1}^{\prime}$, where $x_{1}^{*}$ and $x_{1}^{\prime}$ are the number of customers the primary firm serves in equilibrium with unlicensed and licensed sharing, respectively.

The difference in the profit maximization problem facing the primary SP in the two scenarios is only due to swapping the following two constraints:

with unlicensed sharing: $\quad p_{1}+g\left(x_{1}^{*} / C\right)=P\left(X^{*}\right)$, with licensed sharing: $\quad p_{1}+g\left(x_{1}^{\prime} / C\right)=P(X)$. 
TABLE I

COMPARISON OF IMPACT ON WELFARES IN INVERSE DEMAND FUNCTION PARAMETERS.

\begin{tabular}{c|c|c|c}
\hline & W/O Sharing & unlicensed sharing & licensed Sharing \\
\hline Homogeneous & When $0<C \leq 2 a / b$, & When $0<C \leq a / b$, & When $0<C \leq 4 a / 3 b$, \\
Demand & $S=\frac{b^{2} C}{4}$, & $S=\frac{b^{2} C}{4}$, & $S=\frac{5 b^{2} C}{16}$, \\
& $f_{c}=\frac{b^{2} C}{4}$, & $f_{c}=\frac{b^{2} C}{4}$, & $f_{c}=\frac{5 b^{2} C}{16}$, \\
& $S_{c}=0$. & $S_{c}=0$. & $S_{c}=0$. \\
& When $C>\frac{2 a}{b}$, & When $C>\frac{a}{b}$, & When $C>\frac{4 a}{3 b}$, \\
& $S=a b-\frac{a^{2}}{c}$, & $S=a b-\frac{3 a^{2}}{4 c}$, & $S=a b-\frac{7 a^{2}}{9 C}$, \\
& $f_{c}=a b-\frac{a^{2}}{C}$, & $f_{c}=\frac{a^{2}}{4 C}$, & $f_{c}=\frac{5 a^{2}}{9 C}$, \\
& $S_{c}=0$. & $S_{c}=a b-\frac{a^{2}}{c}$. & $S_{c}=a b-\frac{4 a^{2}}{3 C}$. \\
\hline Heterogeneous & $S=\frac{3 a b^{3} C^{2}+2 a^{2} b^{2} C}{8(a+b C)^{2}}$, & $S=\frac{2 a b^{2} C^{2}+a^{2} b^{2} C}{4(a+b C)^{2}}$, & $S=\frac{9 a b^{2} C^{2}+10 a^{2} b^{2} C}{2(4 a+3 b C)^{2}}$, \\
Demand & $f_{c}=\frac{a b^{2} C}{4(a+b c)}$, & $f_{c}=\frac{a^{2} b^{2} C}{4(a+b c)^{2}}$, & $f_{c}=\frac{5 a^{2} b^{2} C}{4 a+3 b C}$, \\
& $S_{c}=\frac{a b^{3} C^{2}}{8(a+b C)^{2}}$. & $S c=\frac{a b^{2} C^{2}}{2(a+b c)^{2} .}$ & $S_{c}=\frac{9 a b^{2} C^{2}}{2(4 a+3 b C)^{2} .}$ \\
\hline
\end{tabular}
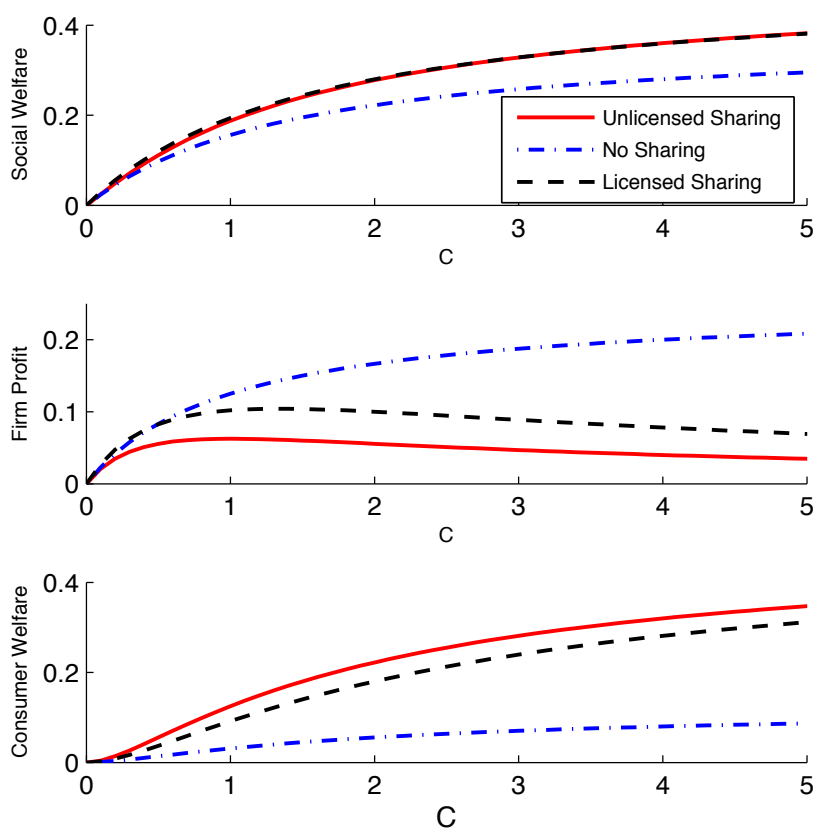

Fig. 5. Comparison of social welfare, firm profit and consumer welfare between unlicensed sharing, no sharing and licensed sharing with linear congestion and linear heterogeneous inverse-demand.

By solving for $p_{1}$ in terms of $x_{1}$ in each of these cases, substituting this into the objective function, $p_{1} x_{1}$, and then setting the derivative equal to zero yields:

$$
\begin{array}{ll}
\text { with unlicensed sharing, } & g\left(\frac{x_{1}^{*}}{C}\right)+\frac{x_{1}^{*}}{C} g^{\prime}\left(\frac{x_{1}^{*}}{C}\right)=P\left(X^{*}\right), \\
\text { with licensed sharing, } & g\left(\frac{x_{1}^{\prime}}{C}\right)+\frac{x_{1}^{\prime}}{C} g^{\prime}\left(\frac{x_{1}^{\prime}}{C}\right)=P(X) .
\end{array}
$$

The left-hand side of these two inequalities is the same function of $x$, which is an increasing function of $x$, since $g(x)$ is increasing, convex. Hence, since $P\left(X^{*}\right) \leq P(X)$, it must be that $x_{1}^{*} \leq x_{1}^{\prime}$.

\section{Conclusions}

We considered a model for price competition with licensed spectrum sharing and showed that it can lead to increased social welfare and consumer welfare compared to no sharing. With limited bandwidth, social welfare and firm profits with licensed sharing are also greater than that obtained under unlicensed sharing, however for larger bandwidths, unlicensed sharing yields greater welfare and firm profits for both approaches vanish. In terms of future work, possible directions include considering sharing in markets with multiple primary firms, each with their own band of spectrum as well as modeling the investment decisions of both primary and secondary providers.

\section{REFERENCES}

[1] Federal Communications Commission, "Unlicensed operation in the TV broadcast bands ; additional spectrum for unlicensed devices below 900 $\mathrm{MHz}$ and in the $3 \mathrm{GHz}$ band" second memorandum opinion and order, 2010.

[2] Federal Communications Commission, "Amendment of the commission's rules with regard to commercial operations in the $3550-3650 \mathrm{MHz}$ band," docket no. 12- 354, Notice of Proposed Rulemaking, Dec. 2012

[3] L. Duan, L. Gao, and J. Huang, "Contract-based cooperative spectrum sharing," IEEE DySPAN, 2011.

[4] R. Berry, M. Honig, V. Subramanian, T. Nguyen, and R. Vohra, "Market Structures for Wireless Services with Shared Spectrum," 2013 Allerton Conference, 2013.

[5] R. Saruthirathanaworakun and J.M. Peha, "Dynamic Primary-Secondary Spectrum Sharing with Cellular Systems,"IEEE Crowncom 2010.

[6] C. Liu and R. Berry, "Competition with Shared Spectrum," 2014 IEEE DySPAN, Apr. 2014.

[7] T. Nguyen, H. Zhou. R. Berry, M. Honig, and R. Vohra, "The impact of additional unlicensed spectrum on wireless services competition," 2012 IEEE DySPAN, pp. 146-155, May 2011

[8] H. Zhou, R. Berry, M. Honig, R. Vohra, "Investment and Competition in Unlicensed Spectrum", 2012 CISS conference, 2012.

[9] D. Acemoglu and A. Ozdaglar, "Competition and Efficiency in congested markets," Mathematics of Operations Research, Feb. 2007.

[10] R. Johari, G. Y. Weintraub and B. V. Roy, "Investment and Market Structure in Industries with Congestion". Operations Research. Sep. 2010.

[11] J. G. Wardrop, "Some theoretical aspects of road traffic research," Proceedings, Institute of Civil Engineers, PART II, vol. 1, pp. 325378, 1952. 